\title{
Acinar Cell Carcinoma with Morphological Change in One Month
}

\author{
Mio Ikeda ${ }^{1}$, Shin Miura ${ }^{1}$, Shin Hamada ${ }^{1}$, Takanori Sano ${ }^{1}$, Ryotaro Matsumoto ${ }^{1}$, Yu Tanaka ${ }^{1}$, \\ Seiji Hongo ${ }^{1}$, Naoki Yoshida ${ }^{1}$, Tetsuya Takikawa ${ }^{1}$, Kazuhiro Kikuta ${ }^{1}$, Kiyoshi Kume ${ }^{1}$, \\ Tatsuo Hata ${ }^{2}$, Michiaki Unno ${ }^{2}$, Yuko Omori ${ }^{3}$, Toru Furukawa ${ }^{3}$ and Atsushi Masamune ${ }^{1}$
}

\begin{abstract}
:
A 64-year-old man was admitted to our hospital to undergo examination of a pancreatic tumor accompanied by sudden epigastric pain. The tumor had a well-defined oval shape that was mostly less enhanced, with the exception of part of the tumor on the pancreatic head side, on contrast enhanced (CE)-CT. However, CECT performed one-month later revealed that the viable part of the tumor grew toward the pancreatic tail with the reduction of necrotic tissue. We performed distal pancreatectomy and the tumor was diagnosed as acinar cell carcinoma (ACC). One important characteristic of ACC is that it may develop morphological changes within a short period of time.
\end{abstract}

Key words: ERCP, EUS, pancreatic cancer, pancreatic tumor

(Intern Med 60: 2799-2806, 2021)

(DOI: 10.2169/internalmedicine.7121-21)

\section{Introduction}

Acinar cell carcinoma (ACC) is a rare pancreatic tumor that accounts for approximately $1-2 \%$ of pancreatic tumors derived from pancreatic acinar cells (1). Typical CT findings of ACC are characterized by an oval shape, clear or indistinct margin, some necrotic components and intraductal tumor plugs $(2,3)$. ACC is often found as a large tumor, with an average size of $8 \mathrm{~cm}(4)$. There is a report of acinarendocrine carcinoma increasing from $1.5 \mathrm{~cm}$ to $7 \mathrm{~cm}$ in a 3.5-year period (5), however, few reports have followed the image findings of ACC. We experienced a case of ACC that showed morphological changes within a short period of time. In this case, the viable portion of the tumor grew toward the pancreatic tail with a reduction of the necrotic component within one month. We recognize this as a valuable case considering the natural history of pancreatic ACC.

\section{Case Report}

The patient was a 64-year-old man who was admitted to a previous hospital due to sudden epigastric pain. His pain spontaneously resolved; however, computed tomography (CT) revealed a pancreatic tumor. He was referred to our hospital for further examination. There were no notable abnormalities in the physical findings. A laboratory examination revealed a slight rise in serum lipase to $75 \mathrm{IU} / \mathrm{L}$. There were no other notable abnormalities, including inflammatory reactions and tumor markers including serum carbohydrate antigen 19-9, carcinoembryonic antigen, S-pancreas-1 antigen, and duke pancreatic monoclonal antigen type 2 . Contrast-enhanced CT (CE-CT) revealed a well-defined oval pancreatic tumor that was mostly less enhanced, with the exception of part of the tumor on the pancreatic head side (Fig. 1). CT did not demonstrate findings suggestive of acute pancreatitis. Magnetic resonance imaging (MRI) showed the tumor with low intensity on T1-weighted imaging, high intensity on T2-weighted imaging, and marked dif-

${ }^{1}$ Division of Gastroenterology, Tohoku University Graduate School of Medicine, Japan, ${ }^{2}$ Department of Surgery, Tohoku University Graduate School of Medicine, Japan and ${ }^{3}$ Department of Investigative Pathology, Tohoku University Graduate School of Medicine, Japan Received: January 19, 2021; Accepted: February 1, 2021; Advance Publication by J-STAGE: March 22, 2021 Correspondence to Dr. Shin Miura, miurashin@med.tohoku.ac.jp 

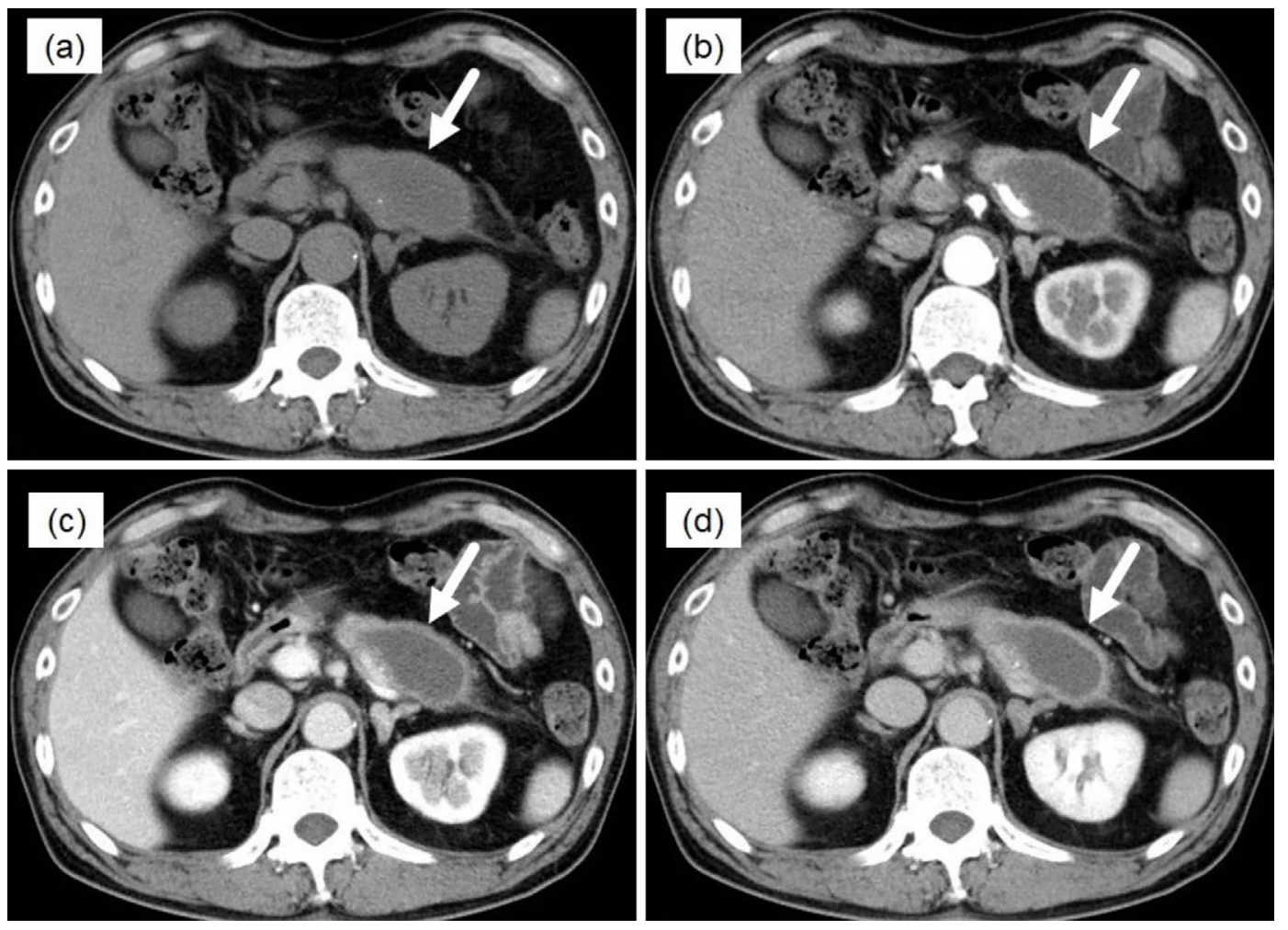

Figure 1. Contrast-enhanced computed tomography. White arrows show the pancreatic tumor. (a) Plain, (b) Early phase, (c) Portal phase, (d) Late phase.
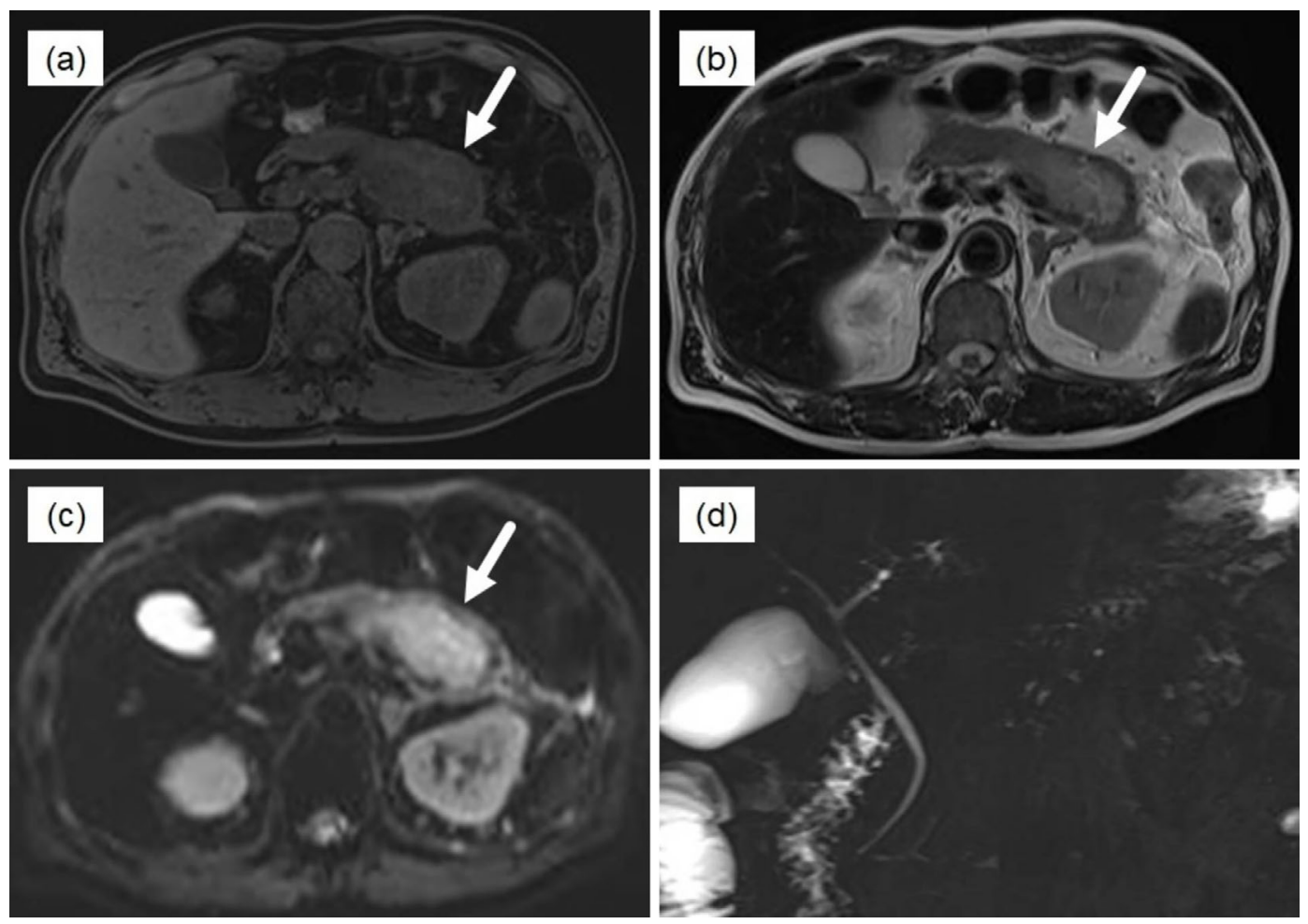

Figure 2. The magnetic resonance imaging. White arrows show the pancreatic tumor. (a) T1WI, (b) T2WI, (c) Diffusion-WI, (d) MRCP.

fusion limitation on diffusion-weighted imaging (Fig. 2). Magnetic resonance cholangiopancreatography (MRCP) did not identify the tail side of the main pancreatic duct (MPD). Endoscopic retrograde cholangiopancreatography (ERCP) re- vealed a filling defect suggesting a tumor plug in the main pancreatic duct of the pancreatic body (Fig. 3). Endoscopic ultrasonography (EUS) showed an oval shaped low-echoic mass (Fig. 4). Contrast-enhanced EUS (CEH-EUS) showed 


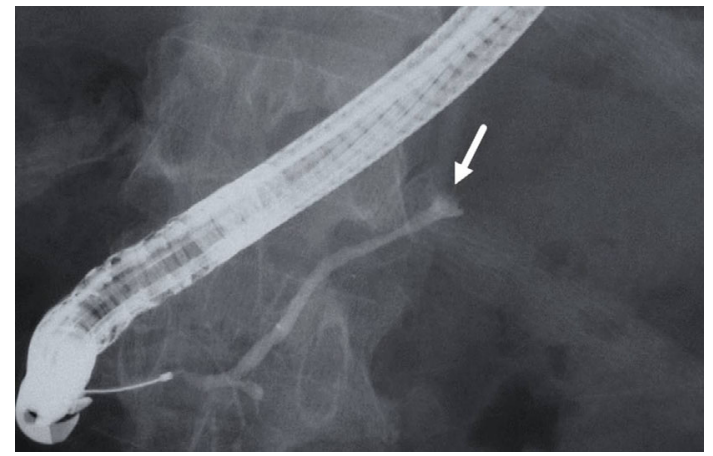

Figure 3. Endoscopic retrograde cholangiopancreatography. White arrow shows the filling defect in the main pancreatic duct. only slight contrast at the edges of the tumor, which was absent in the central area. Fluorodeoxyglucose-positron emission tomography (FDG-PET) revealed the accumulation of FDG limited to the margin of the tumor (Fig. 5). This pancreatic tumor formed an intraductal tumor plug with internal necrosis, and ACC was suspected. We decided to perform distal pancreatectomy.

The accumulation of FDG was also observed in the duodenum on PET-CT (Fig. 5c). However, other modalities showed no abnormalities in the duodenum and we decided to re-perform CE-CT and EUS before surgery. Approximately one month later, CE-CT and EUS revealed that the viable part of the tumor had grown toward the pancreatic tail with a reduction of the necrotic component (Fig. 6, 7).
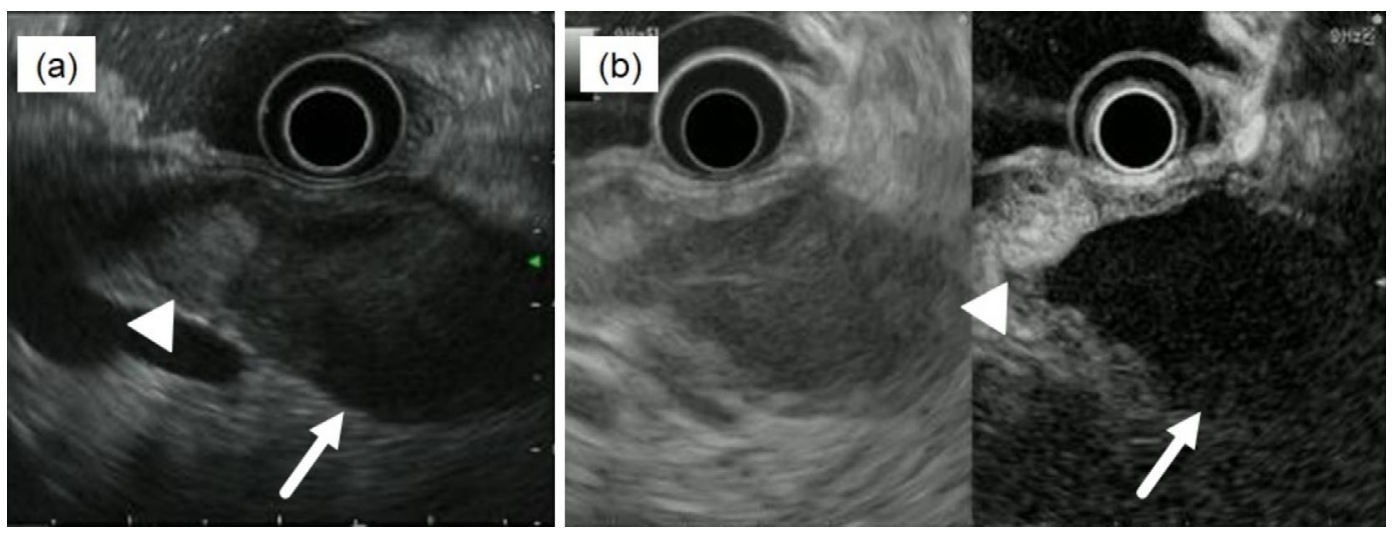

Figure 4. Endoscopic ultrasound. White arrows show the pancreatic tumor. White arrowheads show the viable portion of the tumor. (a) B-mode, (b) Contrast enhanced harmonic image.

(a)

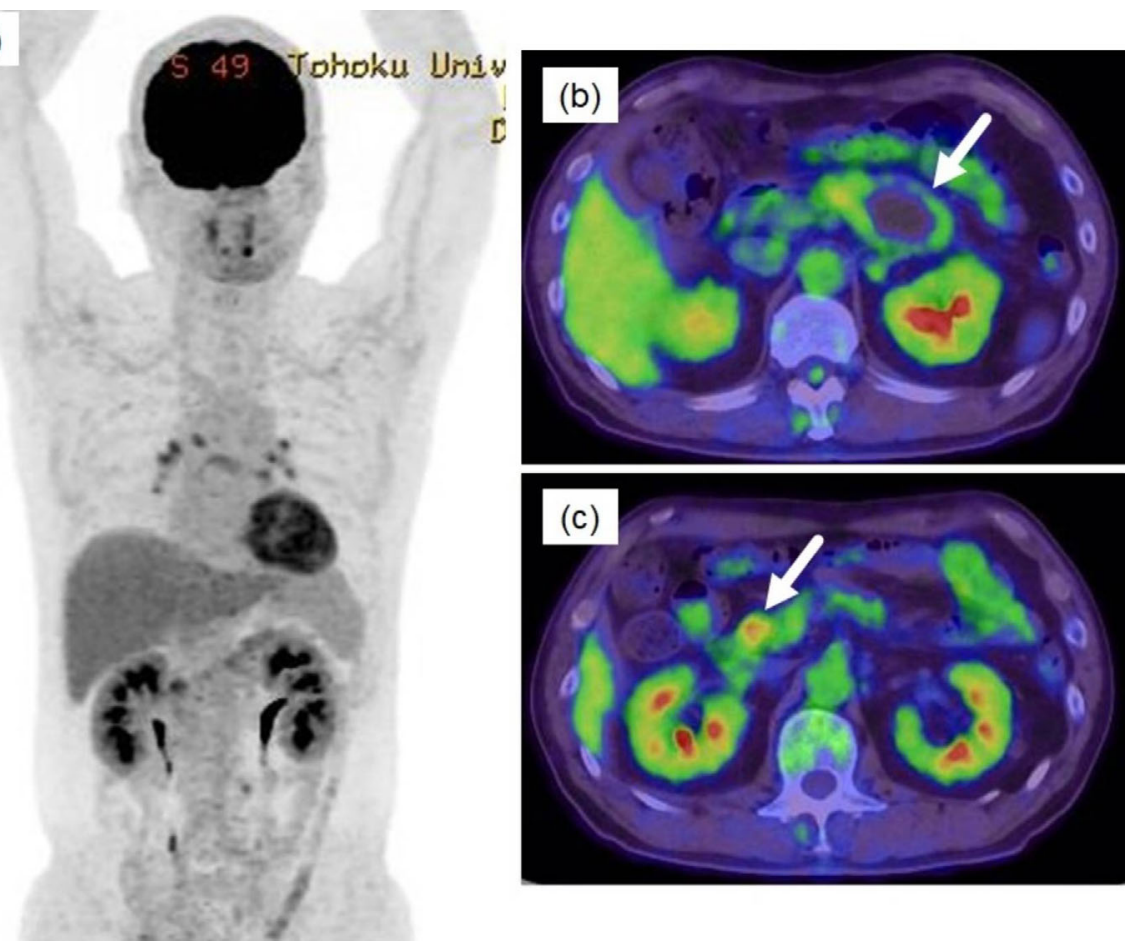

Figure 5. Positron emission tomography-computed tomography. (a) An image of the whole body, (b) The white arrow shows the pancreatic tumor, (c) The white arrow shows the accumulation of FDG in the duodenum. 

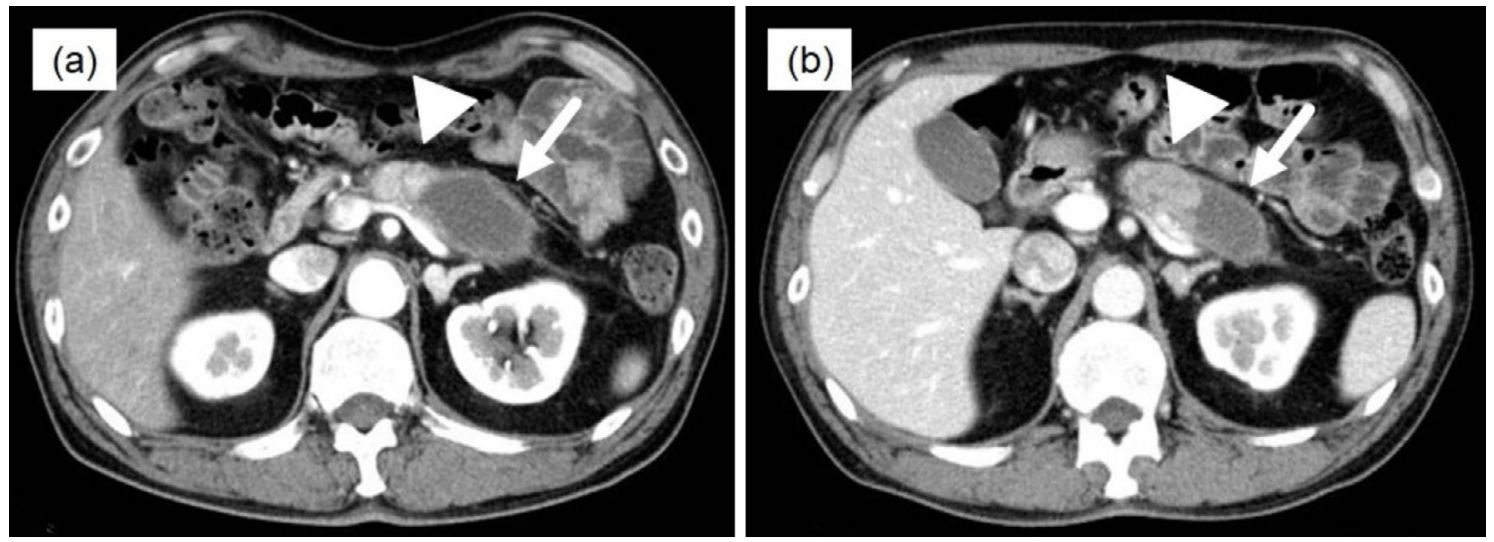

Figure 6. Computed tomography changes after one month. The area without a contrast effect was reduced (arrows), while the viable portion grew from the pancreatic head side toward the tail side (arrowheads) in one month. (a) The initial image, (b) The image obtained one month later.
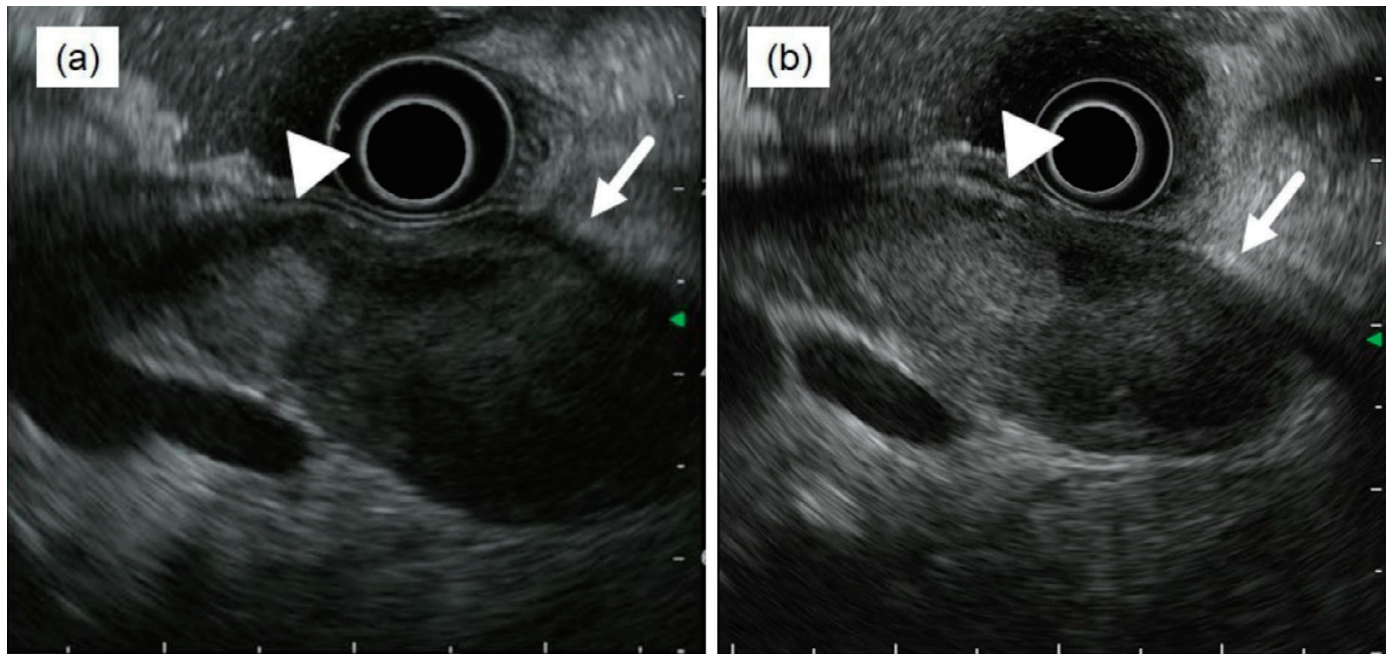

Figure 7. Endoscopic ultrasound changes after one month. EUS showed that the viable portion protruded from the pancreatic head side to the tail side (arrowheads), and the low-echo area decreased (arrows). (a) The initial image, (b) The image obtained one month later.

No abnormalities were found in the duodenum. Thus, we judged this to represent physiological accumulation of FDG. Distal pancreatectomy was performed two months after the first visit.

The postoperative histopathological examination (Fig. 8) revealed that the tumor was $75 \mathrm{~mm}$ in size and was located in the pancreas from the pancreatic tail to the body. On the pancreatic head side, a tumor plug continuing from the tumor developed into the MPD, while the caudal side of the tumor showed extensive necrosis. The tumor also extended into the branch duct of the tail of the pancreas. A pathological examination revealed cuboidal necrotic cells with eosinophilic granular cytoplasm proliferating in acinar form (Fig. 9). An immunohistochemical examination showed that the tumor was positive for BCL-10, trypsin, $\alpha 1$ antichymotrypsin, and $\alpha 1$-antitrypsin. We diagnosed the tumor as pancreatic ACC.
The tumor invaded the peripancreatic tissue and infiltrated the splenic artery. Metastases were found in the two peripheral lymph nodes. The pathological stage was T3N1M0 Stage IIB according to the UICC classification 8th edition (6). He was discharged in good health and did not undergo adjuvant chemotherapy. He has survived for almost two years without recurrence and no abnormalities have been found in the duodenum.

\section{Discussion}

ACC is a rare type of pancreatic tumor, accounting for approximately $1-2 \%$ of all pancreatic tumors. In a previous report, $67 \%$ of ACCs occurred in men at a mean age of 59.6 years (1). ACC often forms a solid mass and shows low contrast effect (2). In addition, ACC is associated with a necrotic component. A previous study on the pathological ex- 


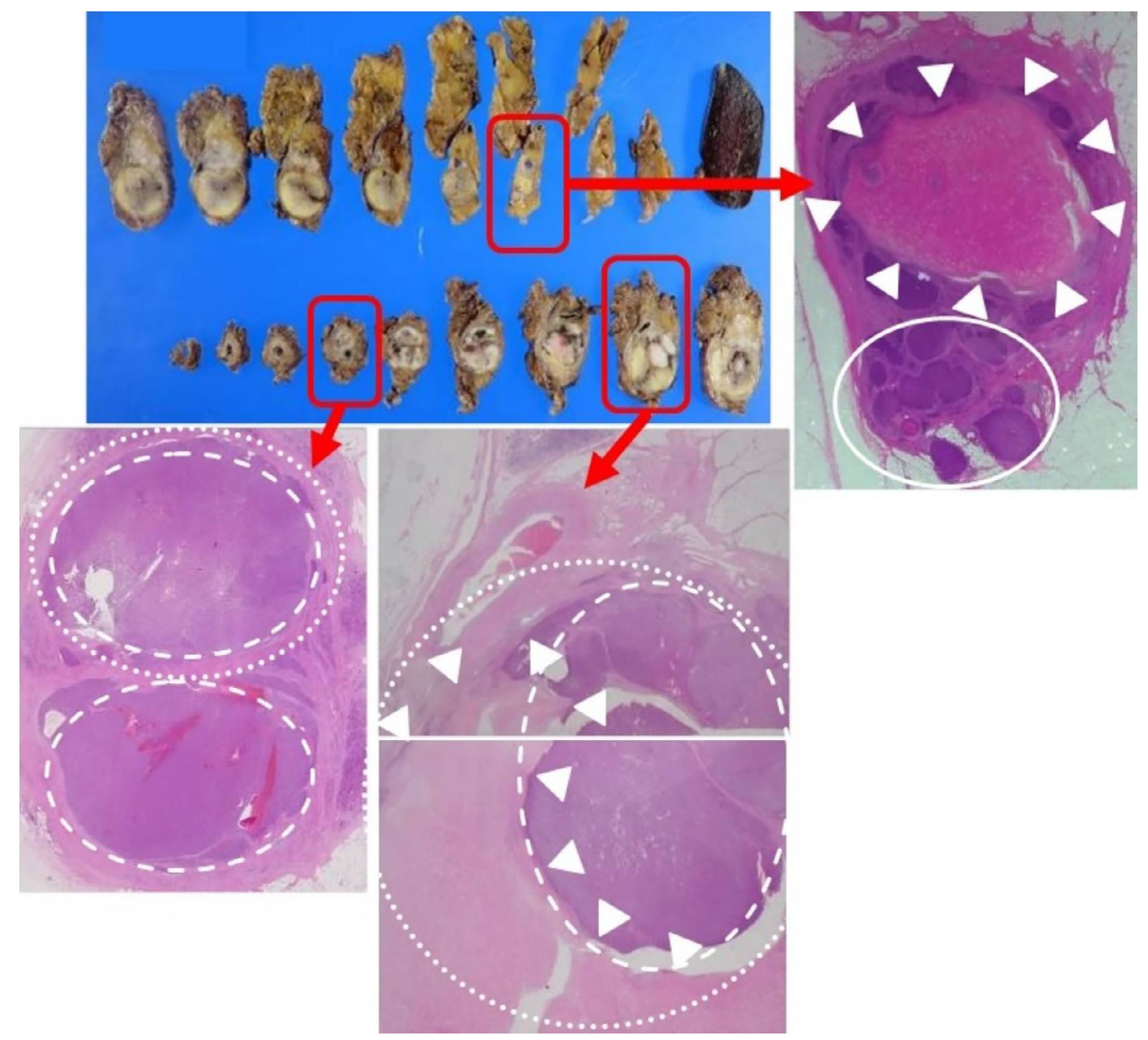

Figure 8. The histopathological findings. The tumor, which measured $75 \mathrm{~mm}$, was located in the pancreatic tail to the body of the pancreas. On the pancreatic head side, a tumor thrombus extended into the main pancreatic duct (dotted line: main pancreatic duct, broken line: tumor). The mass had extensive necrosis (arrowheads). The tumor had spread into the branch duct and invaded the pancreatic parenchyma (solid line).

amination of ACC reported that necrosis was detected in $62 \%$ of ACCs (4). ACC often forms a tumor plug within the main pancreatic duct $(7,8)$. In the present case, the tumor showed internal necrosis and tumor plug within the MPD. These findings are characteristics of ACC. However, no detailed studies have considered how and when internal necrosis is formed. The patient in the present case experienced sudden epigastric pain at the first visit to the previous hospital. Sudden necrosis within the tumor may have caused this symptom. We also experienced a case of ACC with sudden loss of blood flow (9). Therefore, we hypothesized that ACC could undergo morphological changes due to rapid hemodynamic changes. Table presents the 11 cases of ACC or mixed acinar-neuroendocrine tumor that we have experienced. Among these cases, changes in image findings were observed in 3 cases $(27.3 \%)$, and the tumor diameters were 77,25 and $25 \mathrm{~mm}$, respectively. In 2 of the cases, the sudden disappearance of blood flow was observed with abdominal pain. Fig. 10 shows the imaging findings of these two cases in which changes in internal blood flow were ob- served. The reason for the sudden change of blood flow was not clear; however, pathological studies did not reveal arterial occlusion in these three cases, although there were some venous tumor infiltrations. ACC is derived from acinar cells. Thus, it has been hypothesized that autolysis causes necrosis (10). The released pancreatic enzymes induce vascular spasm, which can cause changes in blood flow and necrosis. Vascular spasm due to acute pancreatitis is a well-known phenomenon (11). Mohammadi et al. reported a case of ACC that ruptured spontaneously accompanied by sudden epigastric pain (12). ACC is a tumor that can show morphological changes due to sudden hemodynamic changes, which can be painful. ACC should be included in the differential diagnosis of pancreatic tumors with sudden morphological changes. It is not clear why ACC present sudden hemodynamic changes; thus, further research is needed to elucidate the cause of necrosis.

In the present case, the necrotic component decreased, and the solid component increased from 1.8 to $3.0 \mathrm{~cm}$ after one month. The mean Ki67 labeling index, reflecting high 

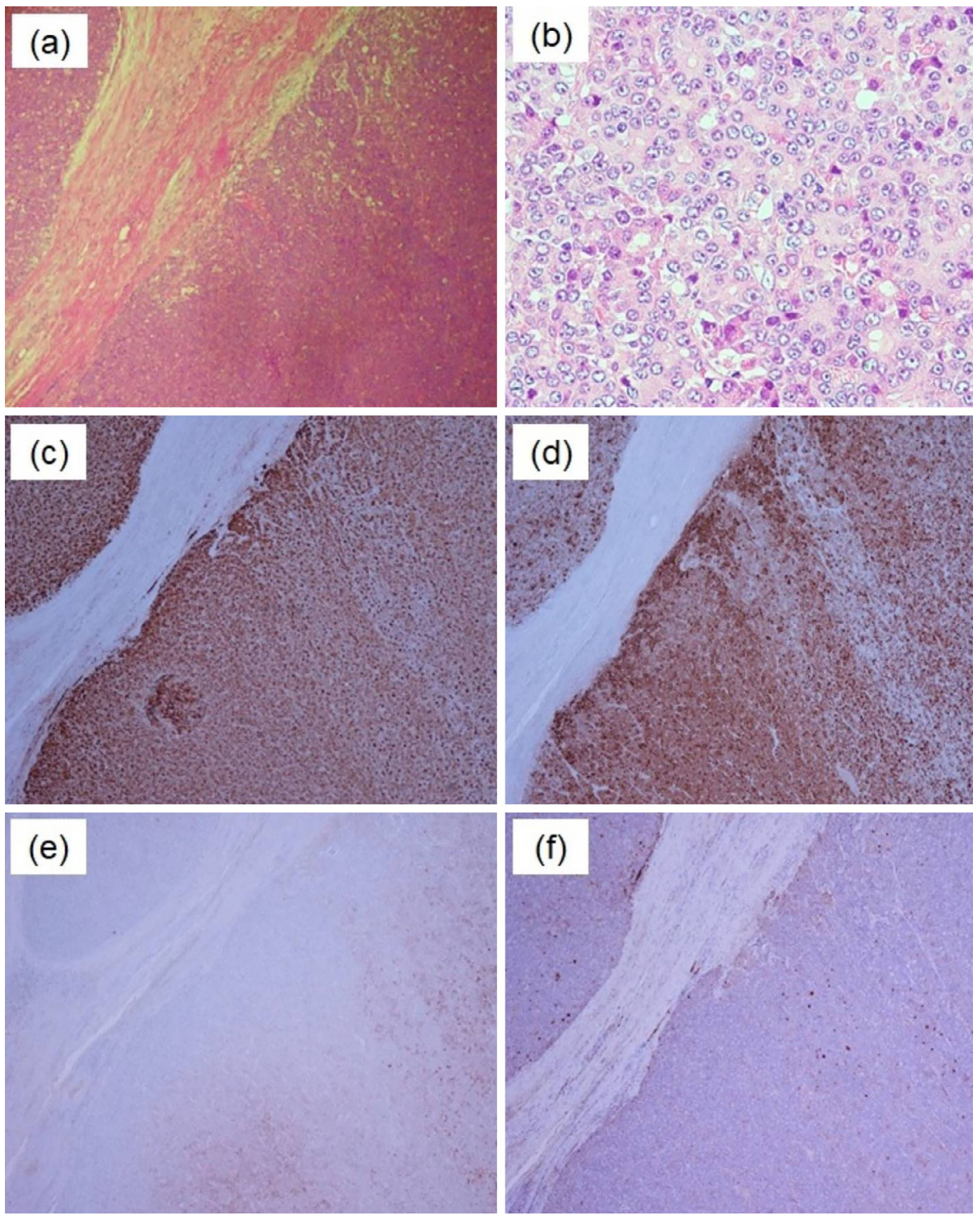

Figure 9. Histopathological findings. (a) Hematoxylin and Eosin (H\&E) staining, $\times 40$, (b) H\&E staining, x400, (c) BCL-10, (d) Trypsin, (e) $\alpha 1$-antitrypsin, (f) $\alpha 1$-antichymotrypsin.

proliferative potential, was $32.21 \%$ in a previous report on ACC (4). The Ki67 index of the present case was 30\%. It was not markedly high and was considered average for ACC. In other words, morphological changes in a short period may not be a rare event in ACC. In a previous study (5), it was reported that acinar-endocrine carcinoma increased from $1.5 \mathrm{~cm}$ to $7 \mathrm{~cm}$ in 3.5 years.

The only curative treatment for ACC is surgery. Wang et al. reported that the median survival period of patients who underwent surgery was 19 months, as opposed to 9 months in cases managed without surgery (13). In this survey, the recurrence rate was reported to be $63.1 \%$. In another report (14), the 1 - and 5-year survival rates were $88 \%$ and
$50 \%$, respectively. In resected cases, the 1- and 5-year survival rates were $92 \%$ and $53 \%$, respectively. The median survival time in resected cases was 61 months. Our patient did not receive postoperative adjuvant chemotherapy. However, no recurrence was detected in the year after surgery. Adjuvant chemotherapy remains controversial, and various regimens were selected depending on the individual case. In a systematic review of ACC (15), adjuvant chemotherapy did not improve the survival rate to a statistically significant extent. However, some reports have described cases of unresectable ACC that showed a good response to chemotherapy, for example, FOLFIRINOX (16), gemcitabine and oxaliplatin (17). We also experienced a case in which S-1 was 
Table. The 11 Cases of Acinar Cell Carcinoma or Mixed Acinar-neuroendocrine Tumor.

\begin{tabular}{|c|c|c|c|c|c|c|c|c|}
\hline No. & Age & Sex & Site & Size & Necrosis & MPD extension & $\begin{array}{l}\text { Changes in } \\
\text { image findings }\end{array}$ & $\begin{array}{l}\text { Accompanied } \\
\text { symptoms }\end{array}$ \\
\hline 1 & 64 & M & tail & $77 \mathrm{~mm}$ & + & + & $\begin{array}{l}+ \\
\text { increase of solid part, } \\
\text { reduction of necrotic tissue }\end{array}$ & $\begin{array}{c}\text { Abdominal pain } \\
\text { (when necrosis occurred) }\end{array}$ \\
\hline 2 & 39 & M & head & $22 \mathrm{~mm}$ & + & - & - & \\
\hline 3 & 60 & $\mathrm{~F}$ & head & $50 \mathrm{~mm}$ & + & - & - & \\
\hline 4 & 53 & M & head & $25 \mathrm{~mm}$ & + & - & $\begin{array}{c}+ \\
\text { sudden disappearance of } \\
\text { the blood flow }\end{array}$ & Abdominal pain \\
\hline 5 & 79 & M & tail & $120 \mathrm{~mm}$ & - & - & - & \\
\hline 6 & 45 & $\mathrm{~F}$ & head & $25 \mathrm{~mm}$ & + & + & $\begin{array}{c}+ \\
\text { sudden disappearance of } \\
\text { the blood flow }\end{array}$ & Abdominal pain \\
\hline 7 & 76 & $\mathrm{M}$ & head & $44 \mathrm{~mm}$ & - & + & - & \\
\hline 8 & 78 & $\mathrm{~F}$ & tail & $40 \mathrm{~mm}$ & - & - & - & \\
\hline 9 & 63 & $\mathrm{~F}$ & head & $34 \mathrm{~mm}$ & - & + & - & \\
\hline 10 & 68 & M & body and tail & $90 \mathrm{~mm}$ & - & - & - & \\
\hline 11 & 88 & M & head & $20 \mathrm{~mm}$ & - & - & - & \\
\hline
\end{tabular}

MPD: main pancreatic duct
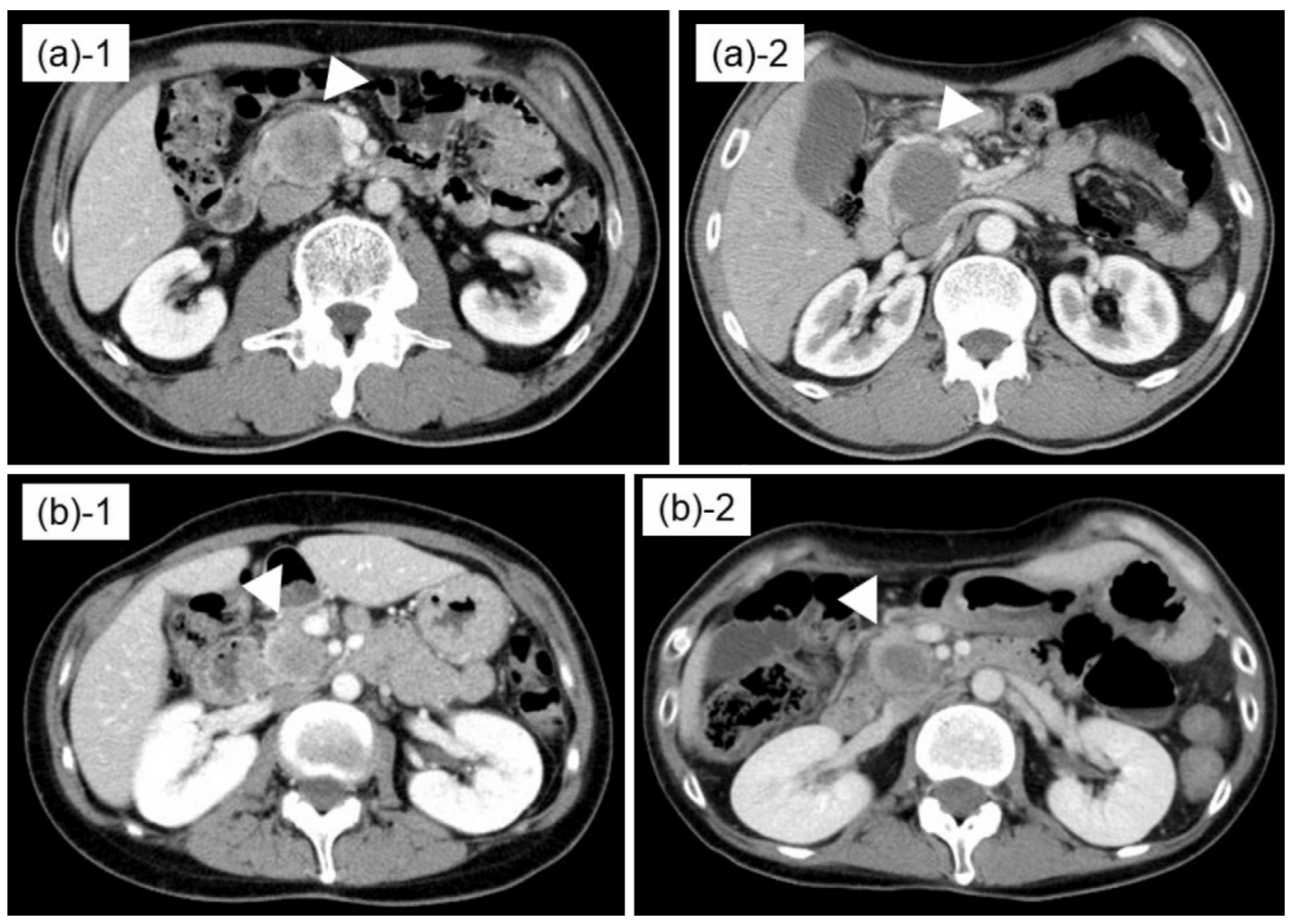

Figure 10. Two cases in which image changes were confirmed during the disease course. (a) Case No. 4 in Table, (a-1) The initial image, (a-2) An image obtained one month later, (b) Case No. 6 in Table, (b-1) The initial image, (b-2) An image obtained one month later.

markedly effective (18).

We reported a case of ACC that showed morphological change within a short period of time. In this case, the viable tumor in the part on the pancreatic head side grew toward the pancreatic tail with a reduction in necrotic tissue after one month. ACC can suddenly develop morphological changes, and such findings may be useful for its diagnosis.
The authors state that they have no Conflict of Interest (COI).

\section{References}

1. Kitagami H, Kondo S, Hirano S, Kawakami H, Egawa S, Tanaka M. Acinar cell carcinoma of the pancreas: clinical analysis of 115 patients from Pancreatic Cancer Registry of Japan Pancreas Soci- 
ety. Pancreas 35: 42-6, 2007.

2. Hu S, Hu S, Wang M, Wu Z, Miao F. Clinical and CT imaging features of pancreatic acinar cell carcinoma. Radiol Med 118: 723731, 2013.

3. Basturk O, Zamboni G, Klimstra DS, et al. Intraductal and papillary variants of acinar cell carcinomas: a new addition to the challenging differential diagnosis of intraductal neoplasms. Am J Surg Pathol 31: 363-370, 2007.

4. La Rosa S, Adsay V, Albarello L, et al. Clinicopathologic study of 62 acinar cell carcinomas of the pancreas: insights into the morphology and immunophenotype and search for prognostic markers. Am J Surg Pathol 36: 1782-1795, 2012.

5. Seki M, Natori K, Kishi Y, et al. Early image findings of acinarendocrine carcinoma of the pancreas: a case report. Suizou 25: 693-701, 2010 (in Japanese, Abstract in English).

6. Brierley JD, Gospodarowicz MK, Wittekind C. TNM Classification of Malignant Tumours. 8th ed. Wiley-Blackwell, Oxford, 2017.

7. Hashimoto M, Matsuda M, Watanabe G, et al. Acinar cell carcinoma of the pancreas with intraductal growth: report of a case. Pancreas 26: 306-308, 2003.

8. Kida A, Matsuda K, Takegoshi K, Matsuda M, Sakai A, Noda Y. Pancreatic acinar cell carcinoma with extensive tumor embolism at the trunk of portal vein and pancreatic intraductal infiltration. Clin J Gastroenterol 10: 546-550, 2017.

9. Kanno A, Masamune A, Hamada S, et al. Sudden disappearance of the blood flow in a case of pancreatic acinar cell carcinoma. Intern Med 2589-2593, 2014.

10. Liu K, Peng W, Zhou Z. The CT findings of pancreatic acinar cell carcinoma in five cases. Clin Imaging 37: 302-307, 2013.
11. Inoue $\mathrm{K}$, Hirota $\mathrm{M}$, Kimura $\mathrm{Y}$, Kuwata $\mathrm{K}$, Ohmuraya M, Ogawa M. Further evidence for endothelin as an important mediator of pancreatic and intestinal ischemia in severe acute pancreatitis. Pancreas 26: 218-223, 2003.

12. Mohammadi A, Porghasem J, Esmaeili A, Ghasemi-Rad M. Spontaneous rupture of a pancreatic acinar cell carcinoma presenting as an acute abdomen. Int J Surg Case Rep 3: 293-295, 2012.

13. Wang $\mathrm{Y}$, Wang S, Zhou X, et al. Acinar cell carcinoma: a report of 19 cases with a brief review of the literature. World J Surg Oncol 14: 172, 2016.

14. Matos JM, Schmidt CM, Turrini O, et al. Pancreatic acinar cell carcinoma: a multi-institutional study. J Gastrointest Surg 13: 1495-1502, 2009.

15. Glazer ES, Neill KG, Frakes JM, et al. Systematic review and case series report of acinar cell carcinoma of the pancreas. Cancer Control 23: 446-454, 2016.

16. Hashimoto M, Hikichi T, Suzuki T, et al. Successful chemotherapy with modified FOLFIRINOX for pancreatic acinar cell carcinoma. Clin J Gastroenterol 10: 564-569, 2017.

17. Ploquin A, Baldini C, Vuagnat $P$, Makhloufi S, Desauw C, Hebbar M. Prolonged survival in a patient with a pancreatic acinar cell carcinoma. Case Rep Oncol 8: 447-450, 2015.

18. Yoshida N, Kanno A, Masamune A, et al. Pancreatic acinar cell carcinoma with multiple liver metastases effectively treated by S-1 chemotherapy. Intern Med 57: 3529-3535, 2018.

The Internal Medicine is an Open Access journal distributed under the Creative Commons Attribution-NonCommercial-NoDerivatives 4.0 International License. To view the details of this license, please visit (https://creativecommons.org/licenses/ by-nc-nd/4.0/).

(C) 2021 The Japanese Society of Internal Medicine

Intern Med 60: 2799-2806, 2021 